\title{
Atomic Scale Study of Point Defects in Graphene using STEM
}

\author{
Wu Zhou, ,** Micah P. Prange, ${ }^{*, * *}$ Mark P. Oxley, ${ }^{* * *}$ Sokrates T. Pantelides, ${ }^{* * *}$ \\ Stephen J. Pennycook, ${ }^{* * * *}$ Jagjit Nanda, ${ }^{* *}$ Chaitanya K. Narula, ${ }^{* *}$ and Juan-Carlos Idrobo ,** \\ *Department of Physics and Astronomy, Vanderbilt University, Nashville, TN 37235 \\ *** Materials Science and Technology Division, Oak Ridge National Laboratory, Oak Ridge, TN \\ 37831
}

Graphene is a fascinating low-dimensional material that has attracted significant attention in recent years [1]. Many interesting phenomena have been observed in this two-dimensional carbon crystal with single atom thickness, e.g. zero-gap semiconductor behavior with ultra-high electron mobility, massless chiral Dirac fermions, and an anomalous quantum Hall effect. Bilayer graphene, in which two layers of graphene stack with certain rotation and/or displacement, is also a subject of intense interest due to its unique properties and promising applications. For example, the band gap of bilayer graphene can be tuned by doping or by applying an electric field perpendicular to the graphene layers, and the optical conductivity of bilayer graphene displays a stacking dependence [2]. From the electron microscopy point of view, graphene and bilayer graphene provide ideal platforms to investigate many fundamental physics and chemistry phenomena via imaging and electron energy loss spectroscopy (EELS).

In this study, we focus on the electron energy low-loss and core-loss spectra acquired from graphene and bilayer graphene at (i) the open and folded graphene edges, (ii) defect sites, and (iii) close to dopant or impurity atoms. EELS data were acquired using a Nion UltraSTEM operated at $60 \mathrm{kV}$, with a $31 \mathrm{mrad}$ probe convergence semi-angle and $48 \mathrm{mrad}$ EELS collection semi-angle. The atomic structure of the graphene samples and the stacking of bilayer graphene were identified by STEM-ADF imaging.

Fig. 1 shows typical STEM-ADF images from several interesting structural features that were used for the EEL spectrum imaging (SI). Fig. 1A was acquired at the edge of the graphene film, where single layer (SL), bilayer (BL), and triple layer (TL) graphene regions can be easily identified from the ADF image intensity. Open and folded graphene edges can often be found on single layer graphene, while the edge of bilayer graphene usually has an open structure that serves as preferred adsorption sites for impurities or heavy dopants. Interesting differences were observed between the EEL spectra taken from the graphene lattice and edge in both low-loss and core-loss regions. Fig. 2A compares the carbon K-edge spectra taken from the graphene lattice and the edge. An additional pre-peak at $281 \mathrm{eV}$ is clearly observed in the spectrum taken from the graphene edge, which is associated with a noticeable decrease of the $\sigma^{*}$ signal. This result is similar to those reported previously [3], however, the pre-peak energy in the present results is red shifted by $1.6 \mathrm{eV}$ with respect to previous reports [3]. The low-loss spectra from the graphene lattice and edge are compared in Fig. 2B. While the peak energy for $\pi$ and $\pi+\sigma$ plasmons are the same in both spectra, a significant decrease in signal intensity and change in shape was observed for the $\pi+\sigma$ plasmon peak taken from the graphene edge.

Impurities are often found in graphene, and they are believed to have a significant effect on the electronic properties [2]. From STEM-ADF imaging, we find that single impurity atoms tend to 
adsorb strongly on dislocation cores in the graphene layer (Fig. 1B). The strong interaction between the impurities and the graphene changes the electronic structure of the graphene layer, which is reflected in the acquired EEL spectra.

A theoretical explanation of the experimental findings will be presented within the framework of total energy first-principles methods based on density functional theory and dynamical electron scattering theory [4].

\section{References:}

[1] A. K. Geim, Science, 234 (2009), 1530.

[2] D. S. L. Abergel et al. Advances in Physics, 59 (2010), 261.

[3] K. Suenage and M. Koshino, Nature, 468 (2010), 1088.

[4] This research was supported by NSF grant No. DMR-0938330 (WZ, JCI), DOE grant DEF002-09ER46554 (MPP, MPO, STP), Oak Ridge National Laboratory's SHaRE User Facility (JCI), which is sponsored by the Office of Basic Energy Sciences, U.S. Department of Energy, and the Office of Basic Energy Sciences, Materials Sciences and Engineering Division, U.S. Department of Energy (SJP).
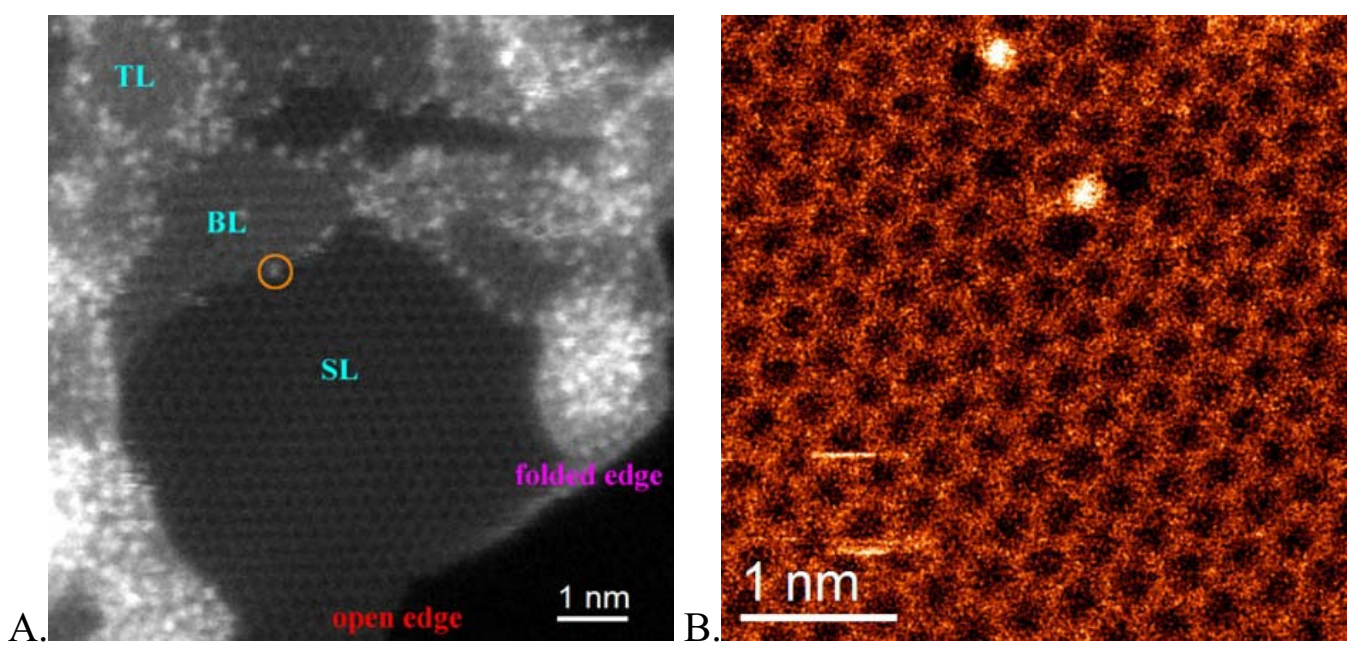

FIG 1. STEM-ADF image of the structural features that were used in the STEM-EELS SI experiments. (A) Various graphene edges; (B) impurities adsorbed on dislocation cores in singlelayer graphene.
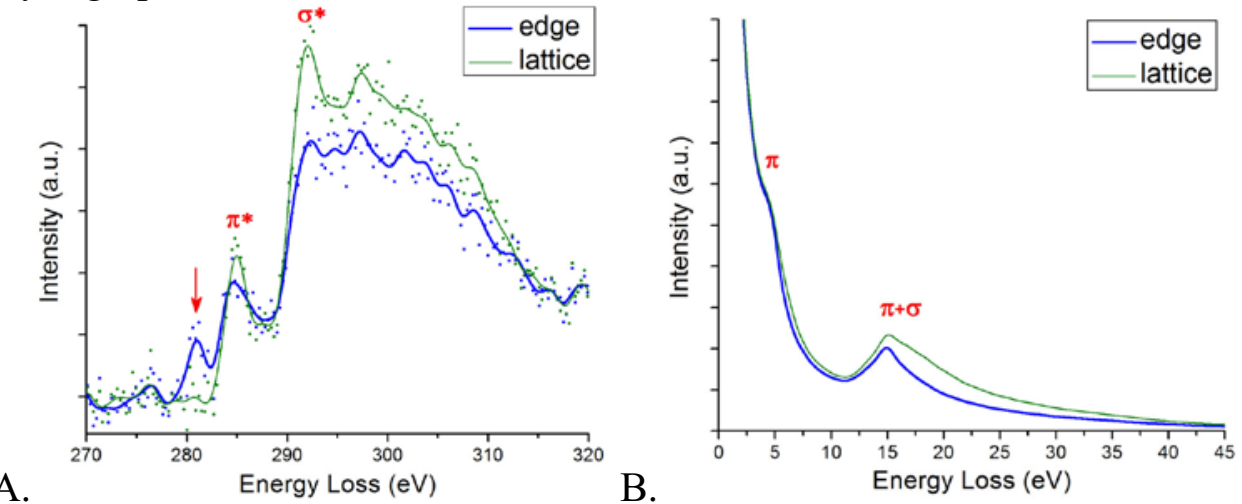

FIG 2. Core-loss (A) and low-loss (B) EELS spectra taken from graphene lattice and edge. Green: graphene lattice; Blue: graphene open edge. 